www.jmscr.igmpublication.org

Impact Factor (SJIF): 6.379

Index Copernicus Value: 71.58

ISSN (e)-2347-176x ISSN (p) 2455-0450

crossref DOI: https://dx.doi.org/10.18535/jmscr/v6i2.92

Journal Of Medical Science And Clinical Research

\title{
Beta-Catenin Expression in Gallbladder Tissues and Its Role in Carcinogenesis and Tumor Progression in Gallbladder Carcinoma
}

\author{
Authors \\ Dr Chandrawati, Dr Santosh Kumar, Dr Shaila Mitra \\ B.R.D.M.C. Gorakhpur
}

\begin{abstract}
Background: Gallbladder carcinoma is the most common malignancy in the biliary tract, the fifth commonest cancer of the gastrointestinal tract. Oncogenes (beta-catenin) has been shown to be involved in the development and progression of gallbladder carcinoma. The present study has been conducted with an aim to study the expression patterns of beta-catenin in different gallbladder tissues to elucidate the role of this oncogene in carcinogenesis and to evaluate its relationship with tumor progression.

Materials and Methods: The study was conducted on histological sections from surgically resected 112 specimens of gallbladder tissue lesions operated at the Department of Surgery, Nehru Chikitsalaya, Gorakhpur which included 66 cases of chronic cholecystitis, 34 cases of gallbladder carcinoma and 12 specimens of gallbladder controls as part of other surgical procedures with no pathology in the gall bladder. Results: Beta-catenin nuclear expression in gallbladder cancer was significantly higher than in chronic cholecystitis and normal gallbladders $(P<0.0001)$. With increasing $(T)$ depth of tumor invasion $(P=$ $0.0231)$ and stage of the lesions $(p=0.0226)$, there is also an increase in the nuclear expression of betacatenin. The level of beta-catenin expression increased from $44.44 \%$ in well differentiated (low grade) to $100 \%$ in poorly differentiated (high grade) tumor $(P=0.0463)$.Thus suggesting a role in tumor progression. Conclusion: In present study, it was observed that expression of beta-catenin get altered in gallbladder carcinoma and has a significant role in carcinogenesis and progression of gallbladder carcinoma.

Keywords: Gallbladder carcinoma, Beta-catenin, "T"- depth of tumor invasion, stage of the lesion.
\end{abstract}

\section{Introduction}

Carcinoma of the gall bladder is the fifth most common tumour in the gastrointestinal tract. Gallbladder carcinoma is a highly lethal disease since it is usually diagnosed at an advanced stage. The 5-year survival rate of patients with gallbladder carcinoma is $\sim 10-30 \%$ despite surgical resection. Moreover, the majority of patients have frequent recurrences following surgery and unsatisfactory results following chemotherapy or radiotherapy ${ }^{1}$.
Gallbladder cancer is reported to be rare in India. However, the incidence of gallbladder cancer in north and central India is very high, it is the commonest gastrointestinal cancer in women.

Carcinogenesis of gallbladder carcinoma is not well understood. Only a limited amount of information is available regarding the molecular carcinogenesis. Oncogenes (beta-catenin) have been shown to be involved in the development and progression of gallbladder carcinoma. ${ }^{2}$ 
Beta-Catenin is a key regulator of the cadherinmediated cell adhesion system, and altered expression and mutation of beta-catenin have been identified in many human malignancies. ${ }^{3}$

$\beta$-catenin nuclear expression is significantly higher in gallbladder carcinoma. Beta-catenin nuclear expression correlates with tumor grade and depth of invasion, thus suggesting a role of this gene in tumor progression of Gallbladder carcinoma. $^{2,4}$

\section{Research Methodology}

Study Design- Cross-sectional study

Project Site - The proposed study was conducted in the Department of Pathology, B.R.D. Medical college, Gorakhpur.

Duration of the Study- during a period from August 2013 to September 2014.

Sample Size- A total of 112 cases were taken for study. The study was conducted on histological sections from surgically resected specimens of gallbladder carcinoma operated at the Department of Surgery, Nehru Chikitsalaya, Gorakhpur which included 66 cases of chronic cholecystitis, 34 cases of gallbladder carcinoma and 12 specimens of gallbladder controls from resections of gallbladder as part of other procedures with no pathology in the gall bladder.

Selection of Cases - Cases were selected on the basis of their final histopathologic diagnosis and their clinicopathologic data were analyzed.

After taking informed consent, histopathological examination and immunohistochemical analysis of beta-catenin expression on all selected gallbladder tissues received in the department was done.

Inclusion Criteria- Clinically suspected cases of benign as well as cancerous lesions of gallbladder were included and patients who agreed to sign on consent form.

Exclusion Criteria- Autolysed sample as well as inadequate sample

\section{Assessment of Beta-Catenin Staining}

- Immunohistochemical staining performed with Polyclonal antibody-
Beta-catenin manufactured by Biogenex, USA.

- Beta catenin expression was judged positive when more than $5 \%$ cells were stained. Staining was evaluated as membranous, cytoplasmic, or nuclear based on the location of staining.

- Stain positive nuclei and membrane Brown

- Counterstained areas- Blue

- Results were scored semi quantitatively.

Standard TNM system of AJCC was used to classify the primary tumors. Tumors were then staged according to the UICC/ AJCC TNM system. $^{5}$

The tumor grades were also noted in each case as Well differentiated (WD), Moderately differentiated (MD), and Poorly differentiated (PD) using criteria by Albores Saavedra. ${ }^{6}$

\section{Data Analysis And Statistical Tools For Observation And Result Of The Study}

Appropriate statistical tools were adopted to do data analysis. Analysis was done by data sorting method and classified by tabulation. The results were scored semi quantitatively and statistical analysis performed.

A statistician's help was sought for interpretation of results. Statistical analysis was done using chi square test to determine the association between two or more than two variables.

Ethical Clearance- The present work was conducted after getting ethical clearance from the institutional ethical committee.

\section{Results}

Observations were made in terms of the relationship of beta-catenin expression with tumor differentiation, invasion, nodal involvement, metastasis and stage of the disease .

A total of 112 cases were taken for present study. Out of 112 cases, 12 cases (10.7\%) were normal (control) gallbladder, 66 cases $(58.9 \%)$ were of chronic cholecystitis and 34 cases (30.3\%) were 
of gallbladder cancer. Table 1, illustrate distribution of cases of gallbladder

Table-1 Distribution of Gallbladder Lesions

\begin{tabular}{|l|c|c|}
\hline $\begin{array}{l}\text { Gallbladder } \\
\text { tissues }\end{array}$ & Number of cases & Percent (\%) \\
\hline $\begin{array}{l}\text { Normal (control) } \\
\text { gallbladder }\end{array}$ & 12 & 10.7 \\
\hline $\begin{array}{l}\text { Chronic } \\
\text { cholecystitis }\end{array}$ & 66 & 58.9 \\
\hline $\begin{array}{l}\text { Gallbladder } \\
\text { cancer }\end{array}$ & 34 & 30.3 \\
\hline \multicolumn{1}{|c|}{ Total } & 112 & $100 \%$ \\
\hline
\end{tabular}

The age of total (112) patients ranged from 21-80 years with a mean and standard deviation of 40.90 \pm 12.18 in chronic cholecystitis cases and $59.12 \pm$ 9.11 in gallbladder cancer cases.

Among chronic cholecystitis lesions, the maximum number of cases, i.e 23 out of 66 cases (34.84\%) were found in age group of 41-50 years followed by 18 cases (27.27\%) in 51-60 years age group.

In gallbladder cancer cases, maximum number of 15 out of 34 cases $(44.11 \%)$ were in the age group of $51-60$ years, followed by $26.47 \%$ in $61-70$ years age group.

Out of 66 benign lesions, the total number of female cases were 53(80.30\%) and 13 cases (19.69\%) were male with F:M ratio of $4: 1$ and out of 34 malignant lesions the total number of female cases were 28 (82.35\%)and 06 cases (17.64\%) were male with F:M ratio of 4.6:1 hence showing the female preponderance in both types of lesions.
Among 66 cases of chronic cholecystitis, 04 cases $(6.06 \%)$ were associated with hyperplasia and 02 cases $(3.03 \%)$ were associated with (pyloric) metaplasia.

All 34 cases of gall bladder carcinoma were Adenocarcinoma, not otherwise specified type. On analysing the tumour grade, out of these 34 cases of Gallbladder adenocarcinoma, 09 cases (26.47\%) were well differentiated, 20 cases (58.82\%) moderately differentiated and, 5 cases $(14.70 \%)$ were poorly differentiated adenocarcinoma. Most common histological grade was moderately differentiated adenocarcinoma $(58.82 \%)$.

On analysing, the depth of tumor invasion, 4 cases $(11.76 \%)$ were categorised as $\mathrm{T} 1,, 13$ cases $(38.23 \%)$ and 16 cases $(47.05 \%)$ were of $\mathrm{T} 2$ andT3 category respectively. 1 case $(2.94 \%)$ was categorised as $\mathrm{T} 4$ according to ' $\mathrm{T}$ ' classification of TNM staging.

When categorized by TNM staging, 4 (11.76\%), $13(38.23 \%), 16(47.05 \%)$, and $1(2.94 \%)$ cases were of T1, T2, T3, and T4 category, respectively. Nodal involvement was seen in 2 cases $(5.88 \%)$, while in 32 cases $(94.11 \%)$ no nodal involvement was present. Metastasis was present in $1(2.94 \%)$ while 33 cases $(97.05 \%)$ had no metastasis. Thus, 4 cases $(11.76 \%)$ had Stage1 disease, 13 cases (38.23\%) had Stage 2 disease, 14 cases $(41.17 \%)$ had stage 3 disease and 3 cases $(8.82 \%)$ were in stage 4.

Table-2 Comparative Evaluation of Beta-Catenin expression in Different Gallbladder Tissues

\begin{tabular}{|l|c|c|c|c|}
\hline Gallbladder tissues & $\begin{array}{c}\text { Gallbladder } \\
\text { cancer(n=34) }(\%)\end{array}$ & $\begin{array}{c}\text { Cholecystitis } \\
(\mathbf{n = 6 6}) \\
(\%)\end{array}$ & $\begin{array}{c}\text { Control } \\
\text { gallbladder }(\mathbf{n}=\mathbf{1 2}) \\
(\%)\end{array}$ & P value \\
\hline $\begin{array}{l}\text { Beta-catenin } \\
\text { expression (nuclear) }\end{array}$ & $\mathbf{2 5 / 3 4 ( 7 3 . 5 2 \% )}$ & $\mathbf{0 / 6 6}(\mathbf{0} \%)$ & $\mathbf{0 / 1 2}(\mathbf{0 \%})$ & $\mathbf{0 . 0 0 0 1}$ \\
\hline
\end{tabular}

Beta-catenin expression was seen in all 34 cases $(100 \%)$ of gallbladder cancer. Expression was heterogeneous, strong in the tumor area and the expression pattern was predominantly membranous and altered to cytoplasmic and focal nuclear in $73.52 \%$ ( 25 of 34 cases). In 66 chronic cholecystitis and 12 control gallbladder specimens, beta-catenin expression was seen in all cases but expression pattern was focal and membranous. While nuclear expression of betacatenin was absent in all chronic cholecystitis and control gallbladder specimens. On statistical 
analysis, beta-catenin expression in gallbladder cancer was significantly higher than in chronic cholecystitis and normal gallbladders ( $\mathrm{P}<$ 0.0001). Thus, beta-catenin expression pattern shifted to nuclear and cytoplasmic with higher staining intensity in gallbladder cancer than chronic cholecystitis and control gallbladder tissues, which showed a focal membranous pattern. (Table-2)

Table-3 Beta-Catenin Expression with Tumor Differentiation Grade in Gallbladder Carcinoma ( $\mathrm{n}=34$ )

\begin{tabular}{|l|cc|cc|}
\hline \multicolumn{1}{|c|}{ Tumor grade } & \multicolumn{2}{|c|}{ Beta -catenin staining positive } & \multicolumn{2}{c|}{ Beta-catenin staining negative } \\
Cases & No. & $(\boldsymbol{\%})$ & No. & $(\boldsymbol{\%})$ \\
\hline Well differentiated $(\mathbf{n = 9 )}$ & $4 / 9$ & $(44.44)$ & $5 / 9$ & $(55.55)$ \\
\hline Moderately differentiated $(\mathbf{n}=\mathbf{2 0})$ & $16 / 20$ & $(80)$ & $4 / 20$ & $(20)$ \\
\hline Poorly differentiated $(\mathbf{n = 5 )}$ & $5 / 5$ & $(100)$ & $0 / 5$ & $(0)$ \\
\hline
\end{tabular}

The level of beta-catenin expression increased from $44.44 \%$ in well differentiated (low grade) to $100 \%$ in poorly differentiated (high grade) tumor.
Thus suggesting a role in tumor progression. (Table-3)

Table-4 Beta-Catenin Expression in TNM Stage of Gallbladder Carcinoma $(\mathbf{n}=\mathbf{3 4})$

\begin{tabular}{|c|c|c|c|c|}
\hline & \multicolumn{2}{|c|}{$\begin{array}{c}\text { Beta -catenin staining positive } \\
\text { Cases }\end{array}$} & \multicolumn{2}{|c|}{$\begin{array}{c}\text { Beta-catenin staining negative } \\
\text { Cases }\end{array}$} \\
\hline & No. & $(\%)$ & No. & $(\%)$ \\
\hline T1 $(n=4)$ & $1 / 4$ & $(25)$ & $3 / 4$ & $(75)$ \\
\hline T2 $(n=13)$ & $8 / 13$ & $(61.53)$ & $5 / 13$ & $(38.46)$ \\
\hline T3 $(n=16)$ & $15 / 16$ & $(93.75)$ & $1 / 16$ & $(6.25)$ \\
\hline T4 $(n=1)$ & $1 / 1$ & $(100)$ & $0 / 1$ & (0) \\
\hline STAGE-1 $(n=4)$ & $1 / 4$ & $(25)$ & $3 / 4$ & $(75)$ \\
\hline STAGE-2 $(n=13)$ & $8 / 13$ & $(61.53)$ & $5 / 13$ & $(38.46)$ \\
\hline STAGE-3 $(n=14)$ & $13 / 14$ & $(92.85)$ & $1 / 14$ & $(7.14)$ \\
\hline STAGE-4 $(n=3)$ & $3 / 3$ & $(100)$ & $0 / 3$ & $(0)$ \\
\hline
\end{tabular}

On analysing the expression of beta-catenin in depth of tumor invasion, with increasing depth of tumor invasion, there is also an increase in the nuclear expression of beta-catenin.
The nuclear pattern of expression of beta-catenin were $25 \%, 61.53 \%, 92.85 \%, 100 \%$ in Stage-1, Stage-2, Stage-3 and Stage-4 respectively. There is increased nuclear staining observed in stage2 and 4 , then stage1. (Table-4)

Table-5 Comparative Evaluation of Beta-Catenin Expression and Adenocarcinoma Differentiation Grades

\begin{tabular}{|c|c|c|c|c|}
\hline $\begin{array}{l}\text { Differentiation } \\
\text { grade }(n=34)\end{array}$ & $\begin{array}{c}\text { Beta-catenin staining } \\
\text { positive } \\
\text { cases }\end{array}$ & $\begin{array}{c}\text { Beta-catenin staining } \\
\text { negative } \\
\text { cases }\end{array}$ & Comparison & P value \\
\hline & No. $\quad(\%)$ & $(\%)$ & & \\
\hline WD $(n=9)$ & $(44.44)$ & $(55.55)$ & \multirow{3}{*}{ WD vs. $M D$ and $P D$} & \multirow{3}{*}{$\mathrm{P}=0.0463$} \\
\hline MD $(n=20)$ & $16 / 20$ & $4 / 20$ & & \\
\hline PD $(n=5)$ & $(100)$ & $0 / 5$ & & \\
\hline
\end{tabular}

WD: Well differentiated adenocarcinoma

MD: Moderately differentiated adenocarcinoma

PD: Poorly differentiated adenocarcinoma

On statistical evaluation, the difference in the level of nuclear expression of beta-catenin between tumor grades (WD vs. MD and PD, taken together) was found to be significant $(\mathrm{P}=0.0463)$. (TABLE-5) 
Table-6 Comparative Evaluation of Beta-Catenin Expression with TNM Stage of Gallbladder Carcinoma

\begin{tabular}{|c|c|c|c|c|c|c|}
\hline & $\begin{array}{r}\text { Beta -ca } \\
\text { posi }\end{array}$ & $\begin{array}{l}\text { n staining } \\
\text { cases }\end{array}$ & $\begin{array}{r}\text { Beta-c } \\
\text { ne }\end{array}$ & $\begin{array}{l}\text { staining } \\
\text { cases }\end{array}$ & Comparison & $\mathbf{P}$ \\
\hline & No. & $(\%)$ & No. & $(\%)$ & & Value \\
\hline T1 $(n=4)$ & $1 / 4$ & $(25)$ & $3 / 4$ & $(75)$ & \multirow{4}{*}{ T1 vs T2-T4 } & \multirow{4}{*}{$\mathrm{P}=0.0231$} \\
\hline T2 $(n=13)$ & $8 / 13$ & $(61.53)$ & $5 / 13$ & $(38.46)$ & & \\
\hline T3 $(n=16)$ & $15 / 16$ & $(93.75)$ & $1 / 16$ & $(6.25)$ & & \\
\hline T4 $(n=1)$ & $1 / 1$ & $(100)$ & $0 / 1$ & $(0)$ & & \\
\hline STAGE-1 $(n=4)$ & $1 / 4$ & $(25)$ & $3 / 4$ & $(75)$ & \multirow{4}{*}{ S1 vs S2-S4 } & \multirow{4}{*}{$\mathrm{P}=0.0226$} \\
\hline STAGE-2 $(n=13)$ & $8 / 13$ & $(61.53)$ & $5 / 13$ & $(38.46)$ & & \\
\hline STAGE-3 $(n=14)$ & $13 / 14$ & $(92.85)$ & $1 / 14$ & $(7.14)$ & & \\
\hline STAGE-4(n=3) & $3 / 3$ & $(100)$ & $0 / 3$ & $(0)$ & & \\
\hline
\end{tabular}

On statistical evaluation of beta-catenin in tumor category of gallbladder carcinoma, the nuclear expression of beta-catenin showed significant difference between $\mathrm{T}$ category $(\mathrm{P}=0.0231)$. Similarly, nuclear expression of beta-catenin showed significant difference with tumor stage $(\mathrm{p}=0.0226)$. (Table-6)

a.

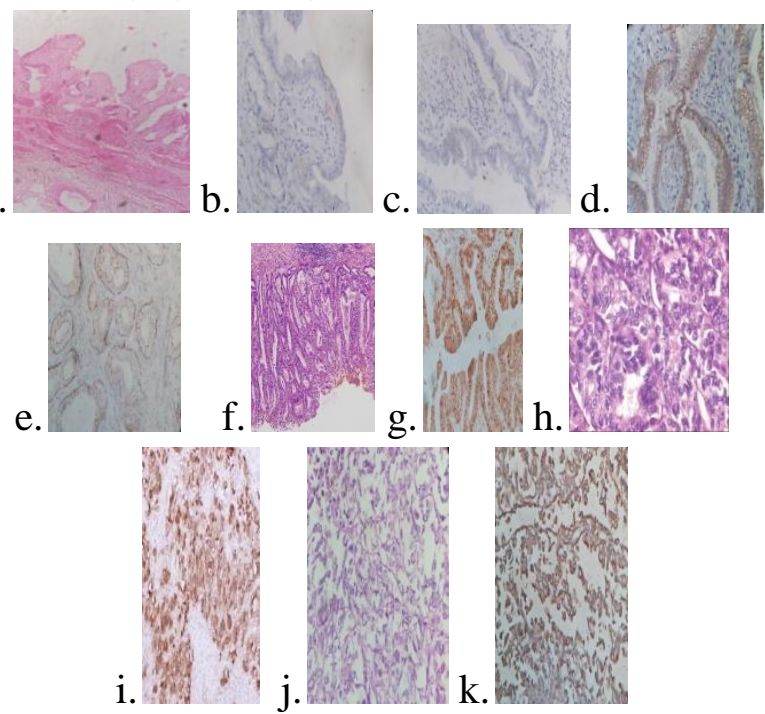

(a) Microphotograph of normal gallbladder showing layers of gallbladder wall (b) same case of normal gallbladder at $400 \mathrm{X}$ showing focal membranous beta-catenin positivity. (c) chronic cholecystitis (400X) showing focal membranous beta-catenin positivity. (d) Chronic cholecystitis with hyperplasia showing beta-catenin membranous positivity. (e) Chronic cholecystitis with pyloric metaplasia showing beta-catenin membranous positivity. (f) $\mathrm{H} \& \mathrm{E}$ stained section (100X) of Well differentiated adenocarcinoma showing large, pleomorphic hyperchromatic nuclei with prominant nucleoli.(g) Same case of Well differentiated adenocarcinoma (400X) showing membranous, cytoplasmic and nuclear beta-catenin positivity. (h) H \& E stained section (400X) showing Moderately differentiated adenocarcinoma with disturbed glandular architecture,large, pleomorphic hyperchromatic nuclei with prominant nucleoli. (i) Same case (400X) of Moderately differentiated adenocarcinoma, showing p53 nuclear positivity. (J) $\mathrm{H} \& \mathrm{E}$ stained section (400X) of poorly differentiated adenocarcinoma with loss of glandular architecture,large, pleomorphic hyperchromatic nuclei with prominant nucleoli.

(k) High power view (400X) of same case showing beta-catenin cytoplasmic and nuclear positivity.

\section{Discussion}

In context to the cases selected for the study, the age of the total 112 patients ranged from 21-80 years. Among benign group, majority of the cases of chronic cholecystitis (23/66 cases, 34.84\%) were found in 41-50 years age group followed by $18 / 66(27.27 \%)$ in 51-60 years age group. Results of previous studies regarding age wise distribution of cases of chronic cholecystitis are shown in table-1.

Table-1 Age wise distribution of cases

\begin{tabular}{|l|c|c|c|}
\hline Age group & $\begin{array}{c}\text { Manuela } \\
\text { Stancu et al } \\
(\mathbf{2 0 0 7 )}\end{array}$ & $\begin{array}{c}\text { Rakesh B.H. } \\
\text { et al } \\
(\mathbf{2 0 1 3})\end{array}$ & Our study \\
\hline $\mathbf{2 1 - 3 0}$ yr & 440 & 12 & 12 \\
\hline $\mathbf{3 1 - 4 0}$ yr & 652 & 12 & 08 \\
\hline $\mathbf{4 1 - 5 0}$ yr & 1111 & 32 & 23 \\
\hline $\mathbf{5 1 - 6 0}$ yr & 740 & 26 & 18 \\
\hline $\mathbf{6 1 - 7 0}$ yr & 440 & 12 & 03 \\
\hline $\mathbf{7 1 - 8 0}$ yr & 236 & 6 & 02 \\
\hline
\end{tabular}

Manuela Stancu et al $(2007)^{7}$, analysed the association of chronic cholecystitis, premalignant 
lesions and gallbladder cancer specimens in 3901 cases of cholecystectomies and observed majority of chronic cholecystitis cases (1111 cases- 30.7\%) in the 41-50 years age group followed by 740 cases (20.4\%) in 51-60 years age group. Rakesh B.H. et al $(2013)^{8}$, reported highest incidence (32\%) of chronic calculous cholecystitis in $5^{\text {th }}$ decade followed by $26 \%$ in $6^{\text {th }}$ decade. Our findings are in accordance with previous studies. R. Thamil Selvi et al (2011) ${ }^{9}$ who studied 65 cases of chronic calculous cholecystitis, and found the highest incidence of these being in the age group of 41-60 years, similar to our study.

Among gallbladder cancer cases, maximum number of cases ( 15 of 34 cases; $44.11 \%$ ) were in the age group of 51-60 years followed by $26.47 \%$ in 61-70 years age group. Majority of cases $(85.29 \%)$ were seen after the age of 50 years.This data is consistent with data in different literature where it has been found that more than $75 \%$ of cases of gallbladder cancer had mean age more than 50 years.

S.Kumar et al $(2014)^{10}$, reported most of the patients (75\%) in the age group of 51-70 years. In the study of Khan RA et al $(2010)^{11}$, the median age of presentation in gallbladder carcinoma was 61 years. Waghmare RS and Kamal RN (2014) ${ }^{12}$, reported gallbladder cancer after 50 years of age in their study.

Results from our study showed that gallbladder cancer is predominantly a disease of females. Out of 66 cases of chronic cholecystitis, 53 (80.30\%) were female and 13 cases $(19.69 \%)$ were male with female to male ratio of $4: 1$ while out of 34 cases of gallbladder cancer, 28 cases $(82.35 \%)$ were females and $06(17.64 \%)$ were males. Male to female ratio was 4.6:1 in gallbladder carcinoma. Incidence of female preponderance in this study are comparable with other studies \{ Gupta SC et al (2000) ${ }^{13}$; Adam R et al (1947) ${ }^{14}$; Glenn F et al (1964) ${ }^{15}$; Khanna R et al (2006) ${ }^{16}$; Aulakh R $(2007)^{17}$; Sayeed Unisa et al (2011) ${ }^{18}$; Randi et al $\left.(2006)^{19}\right\}$. Our findings are in accordance with Santanu Acharyya et al $(2012)^{20}$ and Manuela Stancu et al $(2007)^{7}$ who reported female to male ratio of $4: 1$ in both types of lesions. Giang T H et al (2012) ${ }^{21}$ reported female to male ratio of 4.5:1 in gallbladder carcinoma, similar to our findings. Tyagi et al (1992) ${ }^{22}$, however showed a higher incidence than others with a ratio of 6.5:1.

Most of the available literature shows that the female have been the common victim of gallbladder disease. Why females suffer more is unclear. Some authors have linked oestrogen and sex hormones with gallbladder disease presuming that estrogen metabolism in the liver produces an irritant chemical which causes inflammation and subsequently infection in the biliary passages. Higher and extended exposure to female sex hormones (such as oestrogen and progesterone) may be a main factor.

In the present study, out of total 112 cases, 66 cases $(58.9 \%)$ were chronic cholecystitis while 34 cases $(30.3 \%)$ were gallbladder cancer, representing chronic cholecystitis as the common benign lesion.

Manuela Stancu et al $(2007)^{7}$, analysed 3901 specimens of cholecystectomies and found chronic cholecystitis in 3619 cases $(92.8 \%)$ and gallbladder cancer in 32 cases $(0.8 \%)$ revealing chronic cholecystitis as the commonest lesion. Narang et al (2014) ${ }^{23}$, also reported chronic cholecystitis as the most common lesion in their study of 200 specimens of cholecystectomies.

Mittal R et al (2010) ${ }^{24}$, studied 1312 patients of gallbladder diseases, chronic calculous cholecystitis was seen in 1010 (76.98\%) while cancer was observed in 13 patients $(0.9 \%)$. As mentioned in Sheila Sherlock disease of liver and biliary tract, chronic cholecystitis is the most common gallbladder disease and the same reflection was found in our study. Similarly, Zahrani and Mansoor $(2001)^{25}$ documented chronic cholecystitis $(97 \%)$ as the most common lesion.

Out of 66 cases of chronic cholecystitis, hyperplasia was seen in $6.06 \%$ cases and $3.03 \%$ was associated with pyloric metaplasia. 
Manuela Stancu et al (2007) ${ }^{7}$ reported hyperplasia in 124 cases $(7.8 \%)$ of chronic cholecystitis and 86 cases $(5 \%)$ were associated with metaplasia, predominantly of the pyloric type.

Among malignant lesions, all the 34 cases were reported as Adenocarcinoma, not otherwise specified type, on histopathological examination. Out of these, 34 cases of gallbladder adenocarcinoma, 20 cases $(58.82 \%)$ were moderately differentiated and, 09 cases $(26.47 \%)$ were well differentiated and 5 cases $(14.7 \%)$ were poorly differentiated adenocarcinoma. Most common histological grade was moderately differentiated adenocarcinoma (58.82\%).

Our results are comparable with the study of Veloso MGP et $\mathrm{al}^{26}$; Mittal R et al (2010) ${ }^{24}$ and Waghmore RS et al (2014) ${ }^{12}$. They also found that moderately differentiated adenocarcinoma was the commonest histopathological grade on microscopic examination.

Velosa MGP et al ${ }^{26}$, reported adenocarcinoma, NOS type, in $23 / 24$ cases $(95.83 \%)$ and the majority were moderately differentiated adenocarcinoma. Giang TH et al $(2011)^{21}$, also observed adenocarcinoma, NOS type, in most of the cases in their study.

\section{Beta-catenin expression}

There are very limited studies evaluating the role of beta-catenin in gallbladder cancer.

Beta-catenin mutations are associated with cytoplasmic accumulation of free beta-catenin with subsequent nuclear translocation, thus resulting in nuclear expression pattern. In our study, nuclear expression of beta-catenin was observed in 25 of 34 cases $(73.52 \%)$, while its expression was not seen in controls or chronic cholecystitis. Our results are similar to Ghosh et al $(2013)^{2}$. In their study, nuclear expression of betacatenin was observed in $70 \%$ of cases of gallbladder carcinoma, while none of the cases of chronic cholecystitis and normal gallbladder specimen showed nuclear expression of betacatenin.
In study of, Puhalla et al $(2005)^{28}$, significant differences in expression pattern of beta-catenin between normal, inflamed, and cancerous tissues was also observed similar to our study. The betacatenin membranous expression decreased from cholecystitis and normal to malignant tissue. These results show that beta-catenin may be related with gallbladder carcinogenesis.

Kimura et al $(2003)^{27}$, also reported the similar results, demonstrating that beta-catenin protein expression was seen in both the nucleus and cytoplasm of cancer tissues, while the non- cancerous tissues displayed intense membranous staining. They also found significant correlation between cytoplasmic or nuclear betacatenin immunoreactivity and clinicopathological status of gallbladder carcinoma, especially in the poorer histological differentiation grade $(\mathrm{P}<$ 0.05). They suggested that beta-catenin alteration might be a minor contributor to the development of gallbladder carcinomas through abnormal Wntwingless expression; however, decreased membranous expression of beta-catenin might be correlated to carcinoma progression through loss of cell adhesive function in E-cadherin-catenin fashion.

In the present study, the nuclear expression of beta-catenin showed a significant increase with the grade of tumor, thus suggesting a role in tumor progression ( $\mathrm{P}=0.0463)$. The expression level of beta-catenin increased from $44.44 \%$ in well differentiated tumor (low grade) to $100 \%$ in poorly differentiated (high grade) tumor. With increasing depth of invasion of tumor $(\mathrm{P}=$ $0.0231)$ and tumor stage $(p=0.0226)$, there is also an increase in the nuclear expression of beta catenin .

Our results are comparable with the study of Ghosh et al $(2013)^{2}$. Their study showed significant increase in nuclear expression of betacatenin, with the tumor grade. The expression was $60.1 \%$ in well differentiated tumor (low grade) to $93.33 \%$ in poorly differentiated (high grade) tumor. An increase in the nuclear expression with 
increasing depth of invasion of tumor was also reported in their study.

Moon et al $(2005)^{29}$. also showed that betacatenin was expressed in $80 \%$ of non malignant specimens, exclusively in the cell membrane, while the cancer specimens showed cytoplasmic and/or nuclear staining. Significantly higher betacatenin expressions were present in cancer cells of the invasive front than in the tumor central areas $(\mathrm{P}<0.001)$, and these expressions were significantly $(\mathrm{P}=0.01)$ associated with the invasion depth.

\section{Conclusion}

From the results of present study and the previous available literature, it was observed that expression of beta-catenin altered in gallbladder cancer. Further studies with large number of cases are required especially in higher cancer institutes which also including an analysis of precancerous lesions will give better insight into the role of this gene in carcinogenesis and progression.

\section{References}

1. Park JS, Yoon DS, Kim KS, et al: Analysis of prognostic factors after curative resection for gallbladder carcinoma. Korean J Gastroenterol, 2006; 48: 32-36.

2. Ghosh M, Sakhuja P, Singh S, Agarwal AK. p53 and beta-catenin expression in gallbladder tissues and correlation with tumor progression in gallbladder cancer. Saudi J Gastroenterol 2013;19:34-9.

3. Chang H, Jee C, Kim W. Mutation and altered expression of beta-catenin during gallbladder carcinogenesis. Am J Surg Pathol 2002;26:758-66.

4. Puhalla H, Herberger B, Soleiman A, Filipits M, Laengle F, Gruenberger T, et al. E-cadherin and beta-catenin expression in normal, inflamed and cancerous gallbladder tissue. Anticancer Res 2005;25:4249-54
5. Fleming ID, Cooper JS, Henson DE, Hutter RV, Kennedy BJ, Murphy GP, et al. AJCC cancer staging manual. 5th ed. Philadelphia, New York: Lippincot-Raven; 1997.

6. Albores-Saavedra J, Henson D, Klimstra D. Tumours of the gallbladder, extrahepatic bile ducts and ampulla of vater. Washington, DC: Armed Forces Institute of Pathology; 2000. p. 668.

7. Manuela Stancu et al. Romanian Journal of Morphology and Embryology 2007, 48(4):335-342.

8. Rakesh B , Rajendra G. A prospective clinicopathological study of 50 cases of chronic calculous cholecystitis in the local population: 2013;12;35:6706-6716.

9. R. Thamil Selvi, Pammy Sinha, P.M. Subramaniam, P.G. Konapur, C.V. Prabha. A clinicopathological study of cholecystitis with special reference to analysis of cholelithiasis. International Journal of Basic Medical Science-July 2011, Vol:2, Issue :2.

10. S K umar, Tiwari A . Evaluation of Microsatellite Instability and Apoptosis in Gall Bladder Malignancy from Patients of a Cohort Exposed to Methylisocyanate. J Carcinog Mutagen 2014:5:171.

11. Rizwan Ahmad Khan, Mohammad Amanullah Khan Advanced presentation of Gallbladder cancer: Epidemioclinicopathological study to evaluate the risk factors and assess the outcome 2010;60:217; 2010.

12. Ramesh S Waghmare, Rima N Kamat. Incidental Gall Bladder Carcinoma in Patients Undergoing Cholecystectomy : A Report of 7 Cases, 2014; 62

13. Gupta SC, Mishra V., Singh PA et al. Gall stone and carcinoma gallbladder. Indian $\mathrm{J}$ Pathol Micribiol 2000;43:147-154.

14. Adam R,Stranahan A. Cholecystitis and cholelithiasis; an analytical report of 1,104 
operative cases. Surg Gynecol Obstet 1947; 85:776-84.

15. Glenn F, Frey C. Re-evaluation of the treatment of pancreatitis associated with biliary tract diseases. Ann Surg 1964; 160: 723-36.6

16. Khanna R, Chansuria R, Kumar M, Shukla HS. Histological changes in gallbladder due to stone disease. Indian J Surg 2006; 68:201-4.

17. Aulakh R, Mohan H, Attri AK, et al. Comparative study of serum lipid profile and gallstone disease. Indian $\mathbf{J}$ Pathol Microbiol 2007; 50:308-12. 29.

18. Sayeed Unisa, Palepu Jagannath, Vinay Dhir, Chiranjeeva Khandelwal, Lalatendu Sarangi, and Tarun Kumar Roy. Population - based study to estimate prevalence and determine risk factors of gallbladder diseases in the rural Gangetic basin of North Indian HPB (Oxford). 2011 February; 13(20):117-125.

19. Randi G, Franceschi S, La Vecchia C. Gallbladder cancer worldwide: geographical distribution and risk factors. Int J Cancer 2006; 118:1591.

20. Santanu Acharyya, Amitabha Chakrobarti, Sourav Sau, Partha Dasgupta, Sandip Ghosh, Anjali Majumdar Incidental Finding of Gall Bladder Carcinoma : A Retrospective Study.2012;116-120

21. Tran H Giang, Tran TB Ngoc and Lewis A Hassell. Carcinoma involving the gallbladder: a retrospective review of 23 cases - pitfalls in diagnosis of gallbladder carcinoma .Diagnostic Pathology 2012; 7:10.

22. Tyagi SP, Tyagi N, Maheshwari V, Ashraf SM, Sahoo P. Morphological changes in diseased gallbladder: a study of 415 cholecystectomies at Aligarh. (J Indian Med Assoc.1992 Jul;90(7):178-81.

23. Dr Arathi. NA, Seema Awasthi A, Ashutosh Kumar A .Pathological Profile
Of Cholecystectomies At A Tertiary Centre 2013:2:1;28-38 .

24. Rohin Mittal, Mark Ranjan, Jesudason, Sukria Nayak. Selective histopathology in cholecystectomy for gallbladder disease. Indian J Gastroentrol 2010 , 29 (1):32-36.

25. Zahrani IH, Mansoor I. Gallbladder pathologies and cholelithiasis. Saudi Med J. 2001 Oct;22(10):885-9.

26. Moema Gonçalves Pinheiro Veloso, Daniel Nava Rodrigues. Gallbladder carcinoma: clinicopathological study of 24 cases , 2011; vol.47 no.4 Rio de Janeiro .

27. Kimura Y, Furuhata T, Mukaiya M, Kihara C, Kawakami M, Okita K, et al. Frequent beta-catenin alteration in gallbladder carcinomas. J Exp Clin Cancer Res 2003;22:321-8.

28. Puhalla H, Herberger B, Soleiman A, Filipits M, Laengle F, Gruenberger T, et al. E-cadherin and beta-catenin expression in normal, inflamed and cancerous gallbladder tissue. Anticancer Res 2005;25:4249-54.

29. Moon W, Park H, Lee H, Pai R, Tarnawski A, Kim K, et al. Co-expression of Cox-2, $\mathrm{C}$-Met and beta-catenin in cells forming invasive front of gallbladder cancer. Cancer Res 2005;37:171-6. 